\title{
THE PROPERTIES OF THE INTENSITY- CURVATURE MEASUREMENT APPROACHES: APPLICATIONS IN MAGNETIC RESONANCE IMAGING OF THE HUMAN BRAIN
}

\section{Carlo Ciulla, Ustijana Rechkoska Shikoska, Dimitar Veljanovski and Filip A. Risteski}

Journal of Institute of Science and Technology

Volume 22, Issue 2, January 2018

ISSN: 2469-9062 (print), 2467-9240 (e)

Editors:

Prof. Dr. Kumar Sapkota

Prof. Dr. Armila Rajbhandari

Assoc. Prof. Dr. Gopi Chandra Kaphle Mrs. Reshma Tuladhar

JIST, 22 (2): 19-31 (2018)

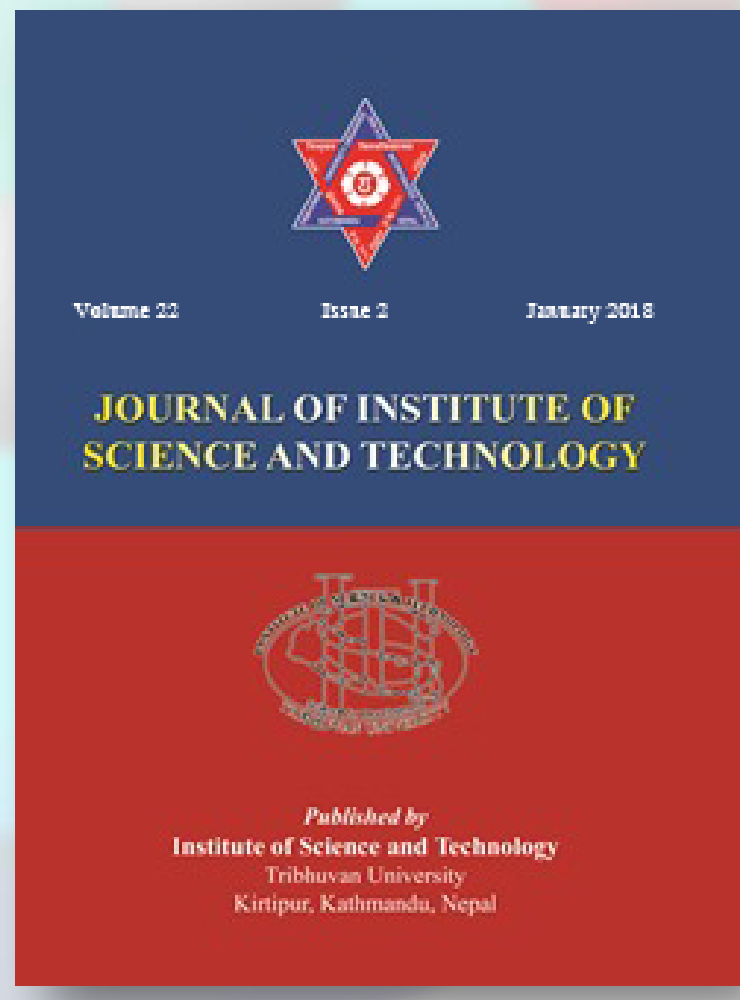

Published by:

Institute of Science and Technology

Tribhuvan University

Kirtipur, Kathmandu, Nepal 


\title{
THE PROPERTIES OF THE INTENSITY-CURVATURE MEASUREMENT APPROACHES: APPLICATIONS IN MAGNETIC RESONANCE IMAGING OF THE HUMAN BRAIN
}

\author{
Carlo Ciulla $^{1^{*}}$, Ustijana Rechkoska Shikoska ${ }^{2}$, Dimitar Veljanovski ${ }^{3}$, and Filip A. Risteski ${ }^{4}$ \\ ${ }^{1,2}$ University of Information Science \& Technology, "St. Paul the Apostle", \\ Partizanska B. B., Ohrid, 6000, Republic of Macedonia \\ ${ }^{3,4}$ Department of Radiology, General Hospital 8-mi Septemvri, Boulevard $8^{\text {th }}$ September, \\ Skopje, 1000, Republic of Macedonia \\ *Corresponding E-mail: carlo.ciulla@uist.edu.mk,cxc2728@njit.edu
}

Received: 21 September, 2017; Revised: 26 October, 2017; Accepted: 1 November, 2017

\begin{abstract}
The intensity-curvature measurement approaches (ICMAs) are re-sampling techniques. The intensitycurvature term is the product between the signal intensity and the classic-curvature and is the root concept at the foundation of the ICMAs. The concept offers six ICMAs: the classic-curvature (CC), the intensitycurvature functional (ICF), the signal resilient to interpolation (SRI), the resilient curvature (RC), the intensity-curvature term before interpolation $\left(\mathrm{E}_{\mathrm{o}}(\mathrm{x}, \mathrm{y})\right)$, and the intensity-curvature term after interpolation $\left(\mathrm{E}_{\mathrm{IN}}(\mathrm{x}, \mathrm{y})\right)$. The ICMAs have the following properties. The CC and the ICF are mask images. The SRI is a filter. The RC is adept to invert, and simultaneously, to smooth and to magnify the grayscale of the image. The aforementioned properties are illustrated with two-dimensional theoretical images and with Magnetic Resonance Imaging (MRI) images of the human brain. The novelty of this work consists of the use of the $\mathrm{E}_{\mathrm{o}}(\mathrm{x}, \mathrm{y})$ and the $\mathrm{E}_{\mathrm{IN}}(\mathrm{x}, \mathrm{y})$ in order to highlight human brain vessels identified with MRI.
\end{abstract}

Keywords: Classic-curvature, Intensity-curvature, Resilient curvature, MRA, Arterial spin-labeling.

\section{INTRODUCTION}

\section{The Magnetic Resonance Imaging Spectrum}

Magnetic Resonance Imaging is the most used noninvasive technique for the study of the human brain and for the diagnosis of the pathologies affecting the central nervous system. Since its inception, MRI has been able to drive research and development efforts in two main directions (Mansfield, 1962; Lauterbur, 1973). One is the development of MRI techniques which have the specific focus on the study of the organ and/or the pathology of interest. The second direction is consequential to the first one because it is driven by the need of assistance of the MRI task at hand, and thus is related to the development of signal-image post-processing techniques. When reviewing MRI allied techniques we are due to mention two important developments: 1) functional Magnetic Resonance Imaging (fMRI) (Ogawa et al., 1993); and Susceptibility Weighted Imaging (SWI) (Haacke et al., 2004). Functional MRI has been used with the specific purpose of studying the human brain function, instead of the anatomy, which is readily possible through the use of MRI. The fMRI signal needs to be post-processed and the development of the fMRI techniques were parallel to the development of software such as the Statistical Parametric Mapping (SPM) (Ashburner, 2012) and the analysis of functional neuroimages (AFNI) (Cox, 1996), among others (Cui et al. 2015). SWI is a technique under current development and its main purpose is to aid the study of the human brain vasculature (Haacke et al., 2004). Technique such as quantitative susceptibility mapping (QSM) nowadays offer methods for the quantification of in vivo iron content, calcifications and changes in venous oxygen saturation (Haacke et al., 2015). MRI on the other hand, has evolved in data acquisition techniques such as: (i) magnetic resonance angiography (MRA), which is used to image the human brain vasculature (Haacke et al., 1999). (ii) 
Diffusion weighted imaging (DWI), which is used to measure the motion of water molecules through the calculation of the diffusion tensor. The diffusion tensor is a measure which includes six independent scalar parameters, from which three eigenvectors are calculated indicating the diffusion direction and three eigen values which are also known to represent the apparent diffusion coefficient (ADC) (Le Bihan et al., 1986, Pierpaoli et al., 1996). (iii) Perfusion weighted imaging (Le Bihan et al., 1986), which is able to measure relative cerebral blood flow, cerebral blood volume, and tissue mean transit time (Cutrer et al., 1998). (iv) Diffusion tensor imaging (DTI), derived from DWI, and used to study, through the apparent diffusion coefficient (ADC), the intra-voxel incoherent motion resulting from molecular diffusion and microcirculation. The intra-voxel incoherent motion is noticeable through the changes in the spin echo relaxation (Le Bihan et al., 1986). (v) Fluid attenuated inversion recovery (FLAIR) MRI, which is a pulse sequence characterized by the suppression of the cerebrospinal fluid (CSF) signal and high T2 weighted imaging, makes it possible to enhance anatomical details of the human brain especially within the context of tumor diagnostics (Hajnal et al., 1992). (vi) MR phase imaging, which uses the phase images obtained at MRI acquisition time, are unwrapped through high pass filtering and thus able to show human brain structures non readily visible through $\mathrm{T} 1, \mathrm{~T} 2$ and/or $\mathrm{T} 2{ }^{*} \mathrm{MR}$ imaging. The capability of the phase images to show brain structures is a direct consequence of the magnetic susceptibility of natural tracers such as deoxyhemoglobin and iron, which can be measured and quantified through the phase (Rauscher et al., 2005; Haacke et al., 2007). (vii) Magnetic Resonance Spectroscopy (MRS), which is able to quantify metabolites in the human brain and thus to increase the diagnostic capability of MRI (Isobe et al., 2010). (viii) Arterial spin-labeling (ASL) Magnetic Resonance Imaging, which is a noninvasive method used to measure in vivo perfusion (Dashjamts et al., 2010).

\section{The intensity-curvature measurement approaches (ICMAs)}

Within the spectrum of MRI post-processing techniques, the ICMAs find their right juxtaposition among the re-sampling techniques (Ciulla et al., 2015). Given a two-dimensional image, it is possible to calculate the ICMAs: the classic curvature $(\mathrm{CC})$, the intensity-curvature functional
(ICF), the signal resilient to interpolation (SRI), the resilient curvature (RC), the intensity-curvature term before interpolation $\left(\mathrm{E}_{\mathrm{o}}(\mathrm{x}, \mathrm{y})\right)$, and the intensity-curvature term after interpolation $\left(\mathrm{E}_{\mathrm{IN}}(\mathrm{x}\right.$, $\mathrm{y})$ ). The classic-curvature is the sum of second order partial derivatives, respect to the spatial coordinates, of the model function fitted to the image data. The intensity-curvature term before interpolation $\left(\mathrm{E}_{\mathrm{o}}(\mathrm{x}, \mathrm{y})\right)$ is calculated as the antiderivative of the product between the value of the signal intensity and the value of the classiccurvature calculated at the origin of the pixel coordinate system (Ciulla et al., 2018). The intensity-curvature term after interpolation $\left(\mathrm{E}_{\mathrm{IN}}(\mathrm{x}\right.$, y)) is calculated as the anti-derivative of the product between the value of the signal calculated from the model function and the classic-curvature calculated at the generic intra-pixel location (Ciulla et al., 2018). The intensity-curvature functional is calculated as the ratio between the $\mathrm{E}_{\mathrm{o}}(\mathrm{x}, \mathrm{y})$ (at the numerator) and the $\mathrm{E}_{\mathrm{IN}}(\mathrm{x}, \mathrm{y})$ (at the denominator). The signal resilient to interpolation is the value of the signal obtained solving the equation between the $\mathrm{E}_{\mathrm{o}}(\mathrm{x}, \mathrm{y})$ and the $\mathrm{E}_{\mathrm{IN}}(\mathrm{x}, \mathrm{y})$. The resilient curvature is the sum of second order partial derivatives of the signal resilient to interpolation, respect to the dimensional variables. The mathematics used to obtain the ICMAs is reported elsewhere (Ciulla et al., 2015). The next section of the paper reports an implicit example. This paper overviews the properties of the ICMAs and presents an application of the $\mathrm{E}_{\mathrm{o}}(\mathrm{x}, \mathrm{y})$ and the $\mathrm{E}_{\mathrm{IN}}(\mathrm{x}, \mathrm{y})$ when studying human brain MRI detected vasculature.

\section{MATERIALS AND METHODS}

\section{Subjects, model functions, ICMAs and MRI acquisitions}

Ten healthy subjects participated in the study. The MRI scanner strength was $1.5 \mathrm{~T}$ and the recording parameters are presented in Table 1. Compliance with the declaration of Helsinki is assured because the MRI scans were collected after proper administration of the informed consent of the patient and in agreement with the ethical committees of Skopje City General Hospital. The model functions fitted to the MRI data for postprocessing were: (i) the bivariate cubic polynomial, (ii) the bivariate cubic Lagrange polynomial, and (iii) the bivariate linear polynomial. Theoretical images were calculated using in house software in order to explore the properties of the ICMAs through the same mathematical procedure that was used to study the MRI images. The MRI data were 
fitted with the polynomial model functions as shown in Table 2. The theoretical images were fitted with the same polynomials, however the cubic and the bivariate cubic Lagrange models were used to calculate the classic-curvature. The intensity-curvature functional was calculated fitting the bivariate linear function.

Table 1: MRI acquisitions parameters.

\begin{tabular}{|l|l|}
\hline MRI acquisitions & $\begin{array}{l}\text { Figures 3a, 3d, 3g, 3j, 3m, } \\
\text { Figures 4a, 4d, 4g, 4j, 4m }\end{array}$ \\
\hline TE & $2.59 \mathrm{msec}$ \\
\hline TR & $7 \mathrm{msec}$ \\
\hline FOV & $280 \times 280$ \\
\hline Pixel matrix size & $410 \times 512$ \\
\hline \# of slices & 9,16 in Figure 4a \\
\hline
\end{tabular}

Table 2: The model polynomial functions fitted to the MRI data.

\begin{tabular}{|l|l|}
\hline $\begin{array}{l}\text { Model polynomial } \\
\text { function }\end{array}$ & ICMA \\
\hline $\begin{array}{l}\text { bivariate cubic } \\
\text { polynomial }\end{array}$ & $\begin{array}{l}\text { classic-curvature }(\mathrm{CC}), \\
\text { intensity-curvature term } \\
\text { before interpolation }\left(\mathrm{E}_{\mathrm{o}}(\mathrm{x},\right. \\
\mathrm{y})), \text { and intensity- } \\
\text { curvature term after } \\
\text { interpolation (E } \mathrm{IN}(\mathrm{x}, \mathrm{y}))\end{array}$ \\
\hline bivariate linear & $\begin{array}{l}\text { intensity-curvature } \\
\text { functional (ICF) }\end{array}$ \\
\hline $\begin{array}{l}\text { bivariate cubic } \\
\text { Lagrange polynomial }\end{array}$ & $\begin{array}{l}\text { signal resilient to } \\
\text { interpolation (SRI), and } \\
\text { resilient curvature (RC) }\end{array}$ \\
\hline
\end{tabular}

\section{Implicit example of the mathematics of the ICMAs}

Let $f(0,0)$ be the signal intensity and $h(x, y)$ be the model polynomial function. Let $\mathrm{h}(\mathrm{x}, \mathrm{y})$ have the property of second order differentiability. The classic-curvature $(\mathrm{CC}(\mathrm{x}, \mathrm{y}))$ is defined as per equation (1). Let $\mathrm{C}(0,0)$ not to be null.

$\mathrm{CC}(\mathrm{x}, \mathrm{y})=\left\{\left(\partial^{2}(\mathrm{~h}(\mathrm{x}, \mathrm{y})) / \partial \mathrm{x}^{2}\right)+\left(\partial^{2}(\mathrm{~h}(\mathrm{x}, \mathrm{y})) / \partial \mathrm{x} \partial \mathrm{y}\right)\right.$ $\left.+\left(\partial^{2}(\mathrm{~h}(\mathrm{x}, \mathrm{y})) / \partial \mathrm{y} \partial \mathrm{x}\right)+\left(\partial^{2}(\mathrm{~h}(\mathrm{x}, \mathrm{y})) / \partial \mathrm{y}^{2}\right)\right\}$

Let the intensity-curvature term before interpolation $\left(\mathrm{E}_{\mathrm{o}}(\mathrm{x}, \mathrm{y})\right)$ be defined as:
$E_{o}(x, y)=\iint f(0,0) \cdot \operatorname{CC}(0,0) d x d y$

Let the intensity-curvature term after interpolation $\left(\mathrm{E}_{\mathrm{IN}}(\mathrm{x}, \mathrm{y})\right)$ be defined as:

$E_{I N}(x, y)=\iint h(x, y) \cdot C C(x, y) d x d y$

Let the intensity-curvature functional $(\operatorname{ICF}(x, y))$ be defined as:

$\operatorname{ICF}(x, y)=E_{0}(x, y) / E_{I N}(x, y)$

Let the signal resilient to interpolation ( $\operatorname{SRI}(\mathrm{x}, \mathrm{y})$ ) be defined as the signal $\zeta(\mathrm{x}, \mathrm{y})$ resulting from the solution in $\mathrm{f}(0,0)$ of the equation between $\mathrm{E}_{\mathrm{o}}(\mathrm{x}, \mathrm{y})$ and $\mathrm{E}_{\mathrm{IN}}(\mathrm{x}, \mathrm{y})$. Because of their definition as per equation (2) and (3), also $\mathrm{E}_{\mathrm{o}}(\mathrm{x}, \mathrm{y})$ and $\mathrm{E}_{\mathrm{IN}}(\mathrm{x}, \mathrm{y})$ are functions of $\mathrm{f}(0,0)$.

$\operatorname{SRI}(\mathrm{x}, \mathrm{y})=\zeta\left(\mathrm{E}_{\mathrm{o}}(\mathrm{x}, \mathrm{y})=\mathrm{E}_{\mathrm{IN}}(\mathrm{x}, \mathrm{y})\right)$

Let the resilient curvature (RC) be defined as:

$\mathrm{RC}(\mathrm{x}, \mathrm{y})=\left\{\left(\partial^{2}(\zeta(\mathrm{x}, \mathrm{y})) / \partial \mathrm{x}^{2}\right)+\left(\partial^{2}(\zeta(\mathrm{x}, \mathrm{y})) / \partial \mathrm{x} \partial \mathrm{y}\right)+\right.$ $\left.\left(\partial^{2}(\zeta(\mathrm{x}, \mathrm{y})) / \partial \mathrm{y} \partial \mathrm{x}\right)+\left(\partial^{2}(\zeta(\mathrm{x}, \mathrm{y})) / \partial \mathrm{y}^{2}\right)\right\}$

\section{RESULTS}

\section{Theoretical Images}

Three theoretical images were calculated in order to explore the Fourier properties of the classiccurvature and the intensity-curvature function. The theoretical images are presented in figures $1 \mathrm{a}, 1 \mathrm{~b}$, 1c. The classic-curvature of the three images was calculated using two polynomial model functions: the bivariate cubic polynomial and the bivariate cubic Lagrange polynomial. The results are presented in figure 1 . The intensity-curvature function was calculated when fitting to the images the bivariate linear function. To date, the $\mathrm{CC}$ and the ICF images can be calculated fitting to the image data a wide array of model polynomial functions, and the most clear and well defined ICF can be obtained fitting the bivariate linear function (Ciulla et al., 2018). The suggestion that CC and ICF images were similar to MRI high pass filtered signal was made through the work reported in Ciulla et al. (2016a). This work further clarifies that both $\mathrm{CC}$ and ICF are different from high pass filtered signal. Indeed, when looking at the images in figure 2, the $\mathrm{k}$-space magnitude of the $\mathrm{CC}$ and the ICF is clearly not the same as the k-space magnitude of the high pass filtered signal. Compare 
figures $2 \mathrm{~d}, 2 \mathrm{e}, 2 \mathrm{f}$ (the $\mathrm{k}$-space magnitude of the high pass filtered theoretical images) versus the images presented in the third, fourth and fifth row from the top. Moreover, figure 2 clarifies that the nature of the $\mathrm{CC}$ and the ICF is not the same (see third, fourth and fifth rows from the top), consistently with their mathematical formulation.
Thus, the concept of intensity-curvature, which merges together the signal intensity value with the sum of the second order partial derivatives calculated respect to the spatial variables, has immediate effect as figure 2 demonstrates. Figure 3 through 7 shows the outcome of the application of the concept in Magnetic Resonance Imaging.
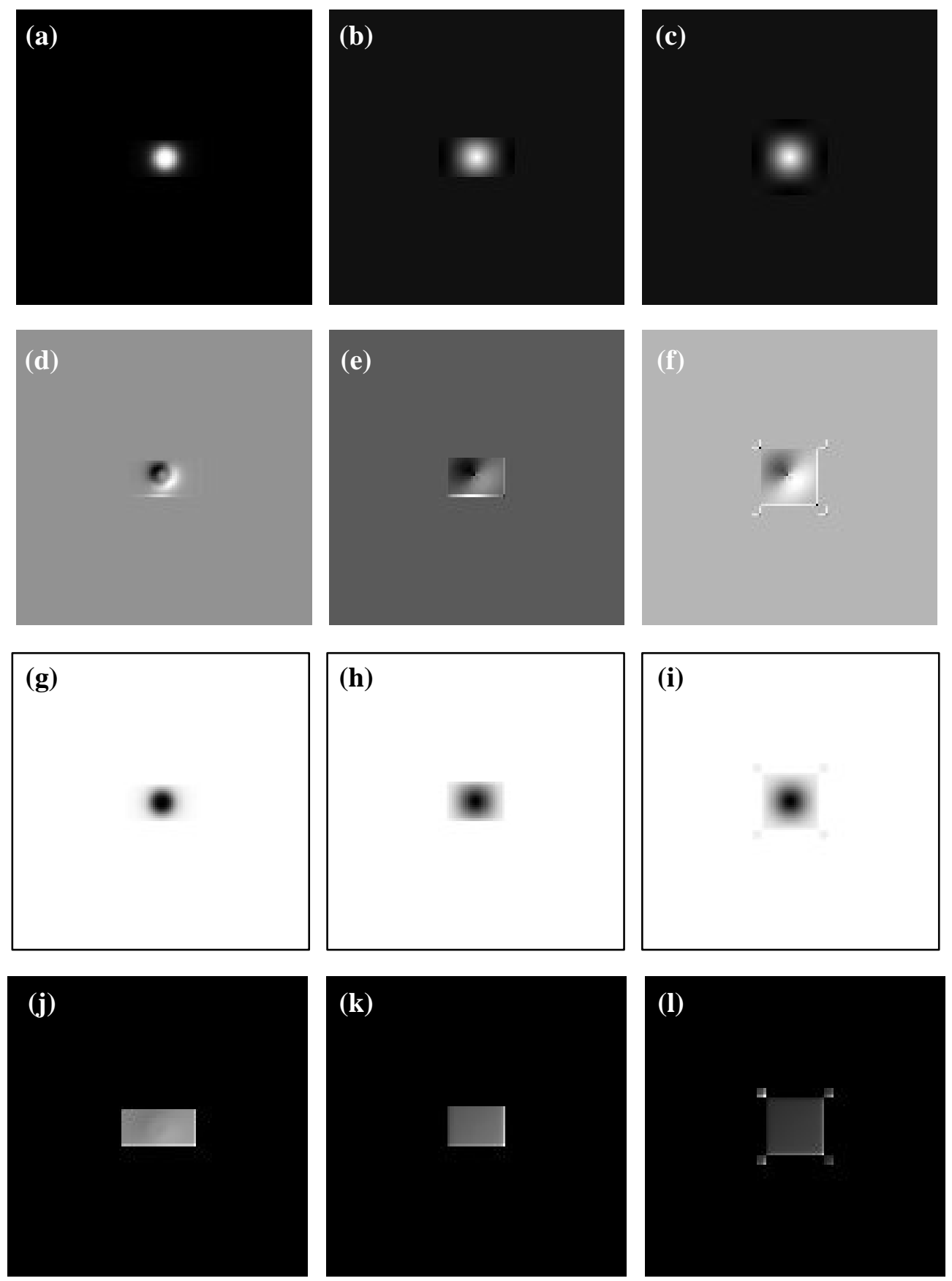

Fig. 1. Theoretical images in (a), (b) and (c). The classic-curvature of the three theoretical images is calculated from the bivariate cubic model function and is presented in $(d),(e)$ and $(f)$. The classiccurvature of the three theoretical images is calculated also with the bivariate cubic Lagrange model function and is presented in $(g),(h)$ and $(i)$. The intensity-curvature functional of the theoretical images is calculated from the bivariate linear function and is presented in $(j),(k)$ and $(l)$. 


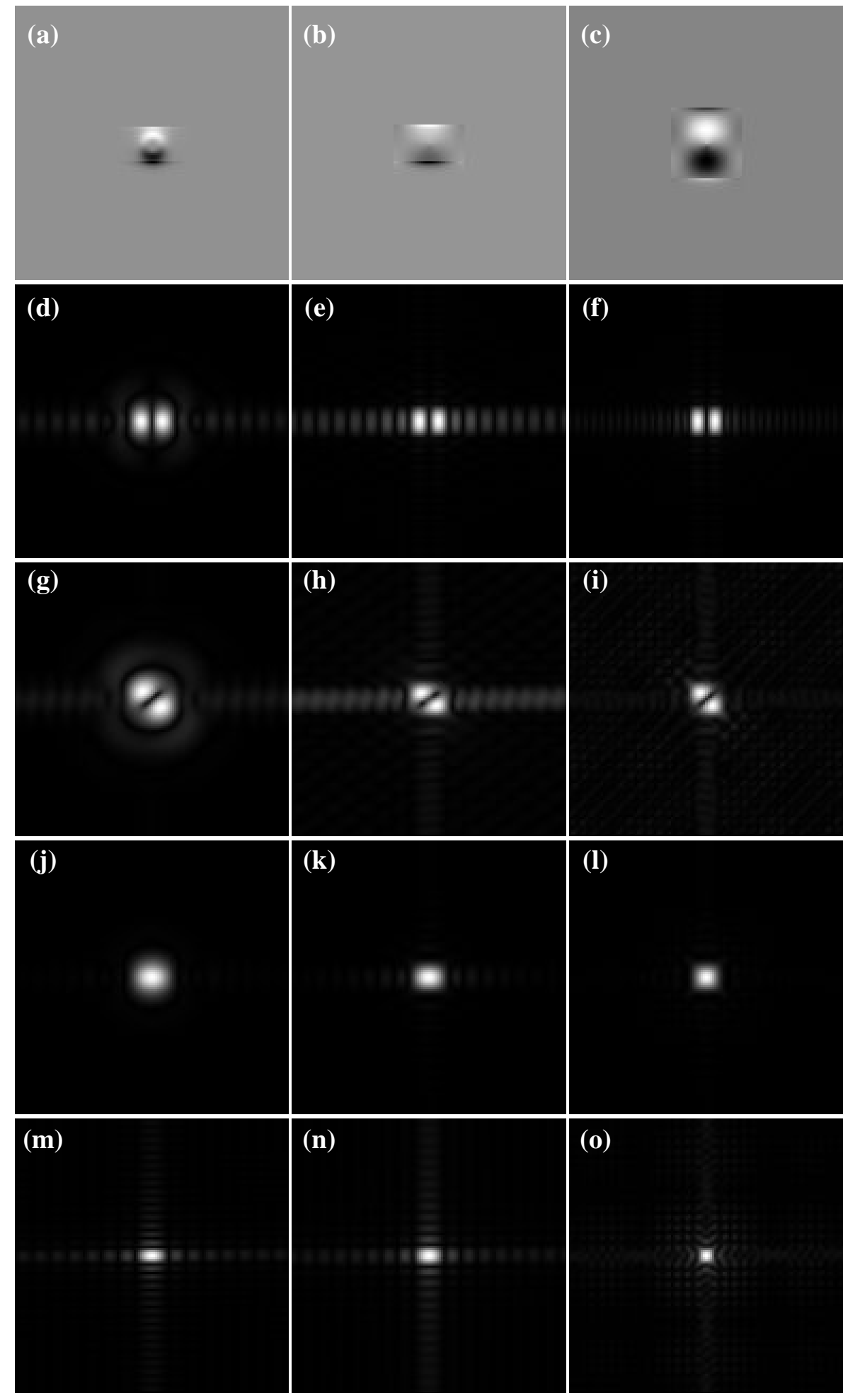

Fig. 2. In (a), (b) and (c) are presented the high pass filtered theoretical images. In (d), (e) and (f) are presented the $k$-space magnitude images of $(a),(b)$ and $(c)$ respectively. The $k$-space magnitude of the classic-curvature images (calculated from the bivariate cubic model function) is presented in the second row from the top (see Figs. 1d, 1e, and 1f). The $k$-space magnitude of the classic-curvature images (calculated from the bivariate cubic Lagrange model function) is presented in the third row from the top (see Figs. $1 \mathrm{~g}, 1 \mathrm{~h}$, and 1i). The $\mathrm{k}$-space magnitude images of the intensity-curvature functional images (Figs. $1 j, 1 k$, and 11$)$ are presented in the bottom row: images in (m), (n) and (o). 


\section{The ICMAs of Magnetic Resonance Images}

This section starts with the presentation of the numerator of the ICF (the intensity-curvature term before interpolation: $\left.\mathrm{E}_{\mathrm{o}}(\mathrm{x}, \mathrm{y})\right)$, and the denominator of the ICF (the intensity-curvature term after interpolation: $\left.\mathrm{E}_{\mathrm{IN}}(\mathrm{x}, \mathrm{y})\right)$. The MRI in figure 3 and 4 is presented to the far left of each row of images, the $\mathrm{E}_{\mathrm{o}}(\mathrm{x}, \mathrm{y})$ is placed in the middle, and the $\mathrm{E}_{\mathrm{IN}}(\mathrm{x}$, $y)$ is presented on the far right of each row. Fig. 3 and 4 present the following two points of discussion. 1) The novelty reported by this paper. The MRI images were selected in the regions of interest showing human brain vasculature. The intensity-curvature terms $\left(\mathrm{E}_{\mathrm{o}}(\mathrm{x}, \mathrm{y})\right.$ and $\left.\mathrm{E}_{\mathrm{IN}}(\mathrm{x}, \mathrm{y})\right)$ were calculated. 2) The two intensity-curvature terms (ICTs) appear to be very much alike because the intensity-curvature functional calculated from the bivariate cubic model polynomial function is almost equal to ' 1 ' across the full spatial extent of the MRI image and thus is unusable. The novelty of the works herein reported can be summarized in three main points: (i) the ICTs display the visually perceptible third dimension (Ciulla et al., 2016a) likewise the classic-curvature when calculated fitting to the MRI data the bivariate cubic model polynomial function (as shown in this paper), (ii) the visually perceptible third dimension might be related to the accumulation of fluids in proximity of the vessels, and (iii) the $\mathrm{k}$-space magnitude of the ICTs is different from the $\mathrm{k}$-space of the high pass filtered signal (as it shall be seen in figure 7), hence the nature of the ICTs is not high pass filtered signal. As far as regards, the second point of discussion, clearly, the use of the intensitycurvature terms replaces the use of the unusable intensity-curvature functional (ICF). The behavior of the ICF in such case is not uncommon and has been observed when fitting also other model polynomial functions. To elucidate the behavior of the images presented in figures 5 and 6 , the following three points can be made. 1) The classiccurvature highlights the vasculature of the human brain showing the visually perceptible third dimension in proximity of the vessels. 2) The signal resilient to interpolation is not a duplication of the original MRI because it behaves as a narrow filter (Ciulla et al., 2016b). 3) The resilient curvature presents the properties of inverting, smoothing and magnifying (in one pass) the grayscale of the MRI (Ciulla et al., 2016b). Figure 7 clarifies the neat difference existing between the high pass filtered MRI image and the $E_{0}(x, y)$ and the $E_{\mathrm{IN}}(\mathrm{x}, \mathrm{y})$. Such difference was documented for the classiccurvature and the intensity-curvature functional, through the k-space magnitude of the theoretical images presented in the section 4.1 titled: 'The Theoretical Images', and is remarked here through the outward show of the high pass filtered MRI image and the outward show of the k-space magnitude of the $\mathrm{E}_{\mathrm{o}}(\mathrm{x}, \mathrm{y})$ and the $\mathrm{E}_{\mathrm{IN}}(\mathrm{x}, \mathrm{y})$ (see (f) versus $(g)$ and $(h))$. Therefore, Fig. 7 clarifies that the meaning and the nature of the intensitycurvature terms $\mathrm{E}_{\mathrm{o}}(\mathrm{x}, \mathrm{y})$ and $\mathrm{E}_{\mathrm{IN}}(\mathrm{x}, \mathrm{y})$ is not high pass filtered signal (likewise the classic-curvature and the intensity-curvature functional).
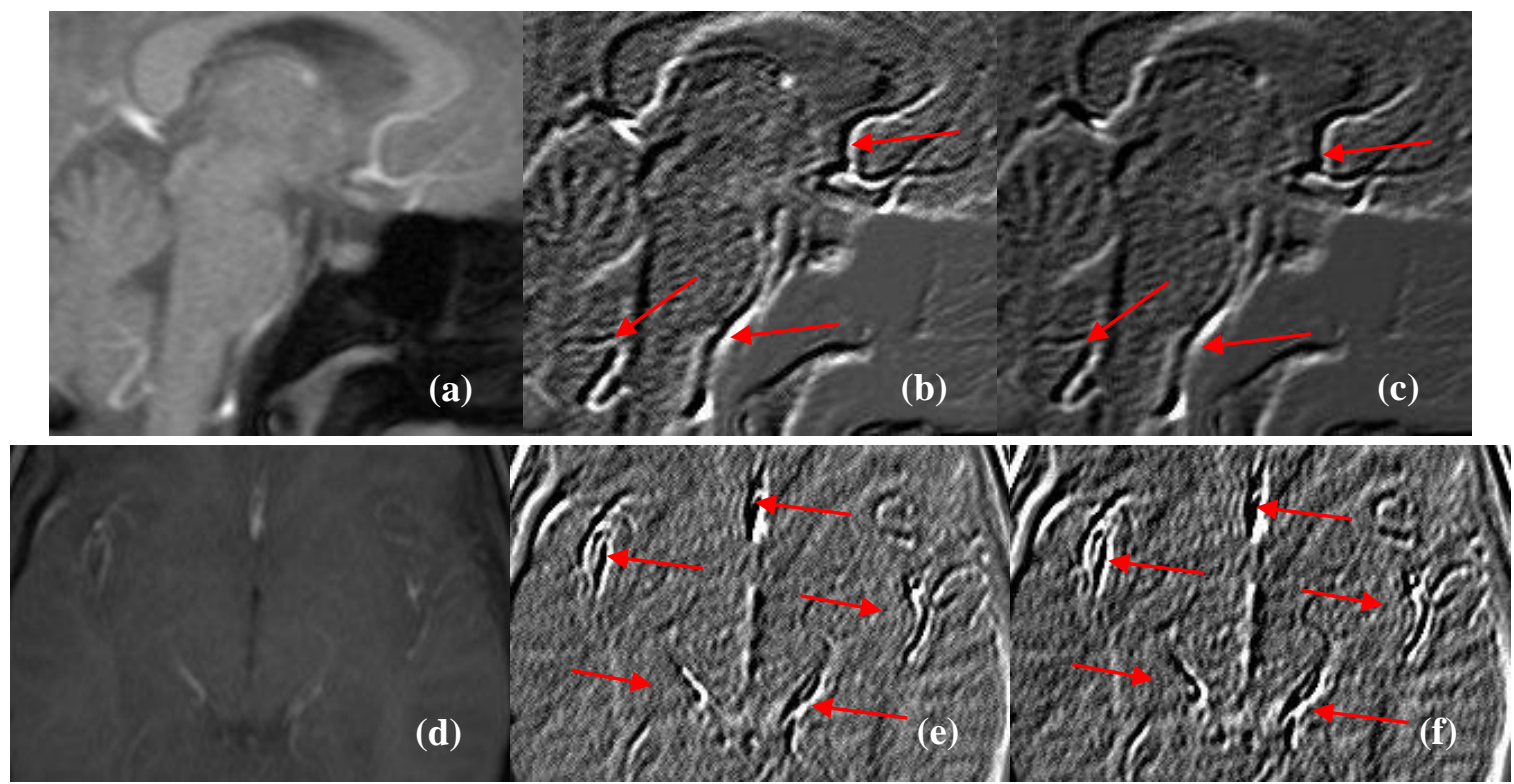


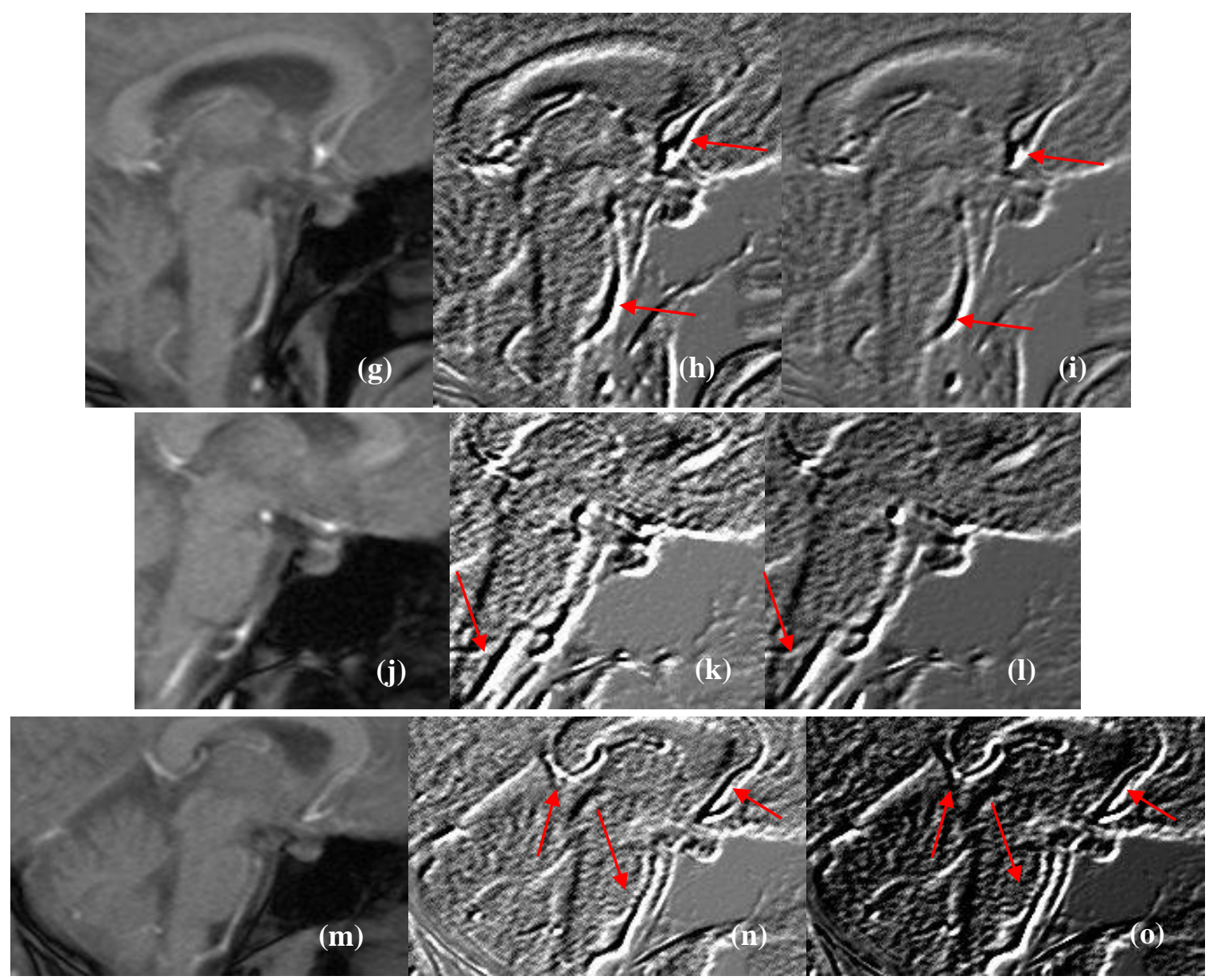

Fig. 3. Magnetic Resonance Imaging (MRI) of regions of interest of the human brain are presented in $(a),(d),(g),(j)$ and $(m)$. The intensity-curvature term before interpolation $\left(E_{o}(x, y)\right)$ is presented in $(b)$, $(e),(h),(k)$ and $(n)$. The intensity-curvature term after interpolation $\left(E_{I N}(x, y)\right)$ is presented in $(c),(f),(i)$, (l) and (o). The intensity-curvature terms were calculated using the bivariate cubic model function fitted to the MRI data. The outward show of the two ICTs is similar because the ratio between the $E_{o}(x, y)$ and the $E_{I N}(x, y)$ is very close to ' 1 '. The arrows point to the human brain vessels.

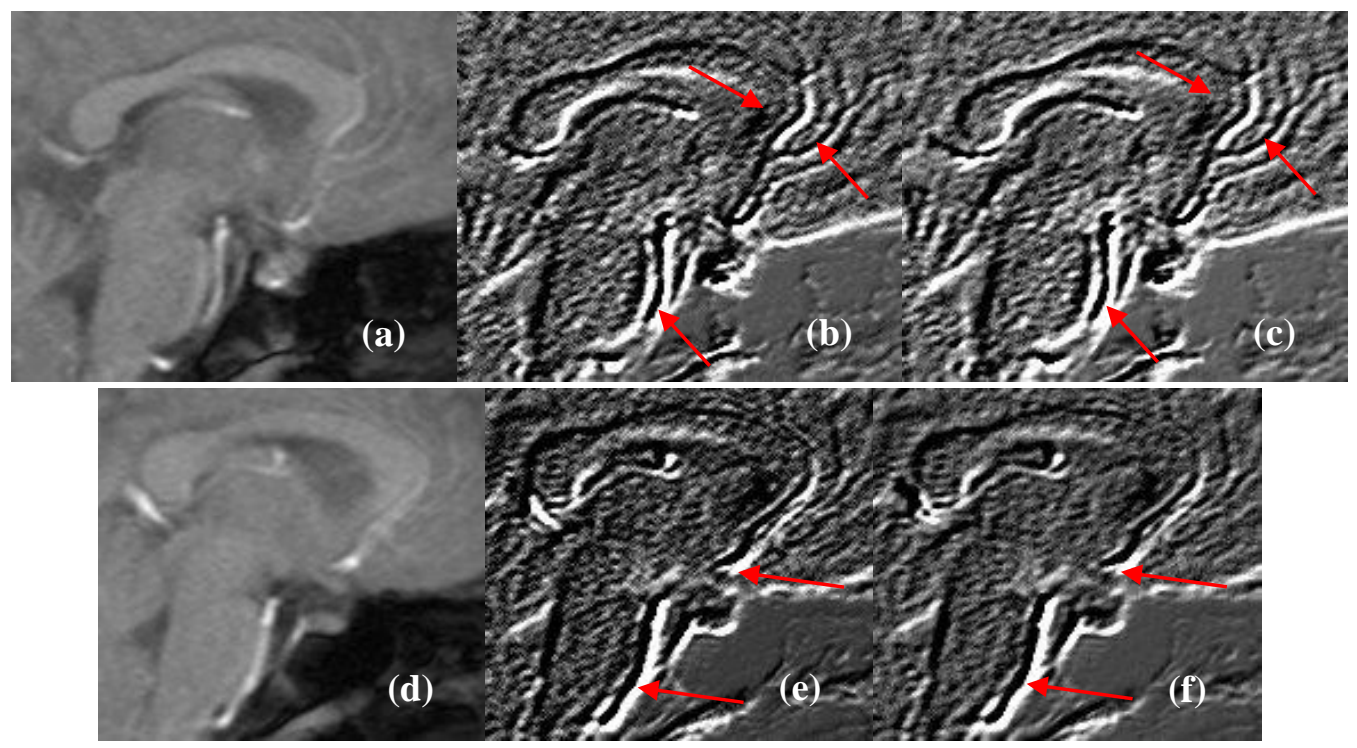


The Properties of the Intensity-Curvature Measurement Approaches...

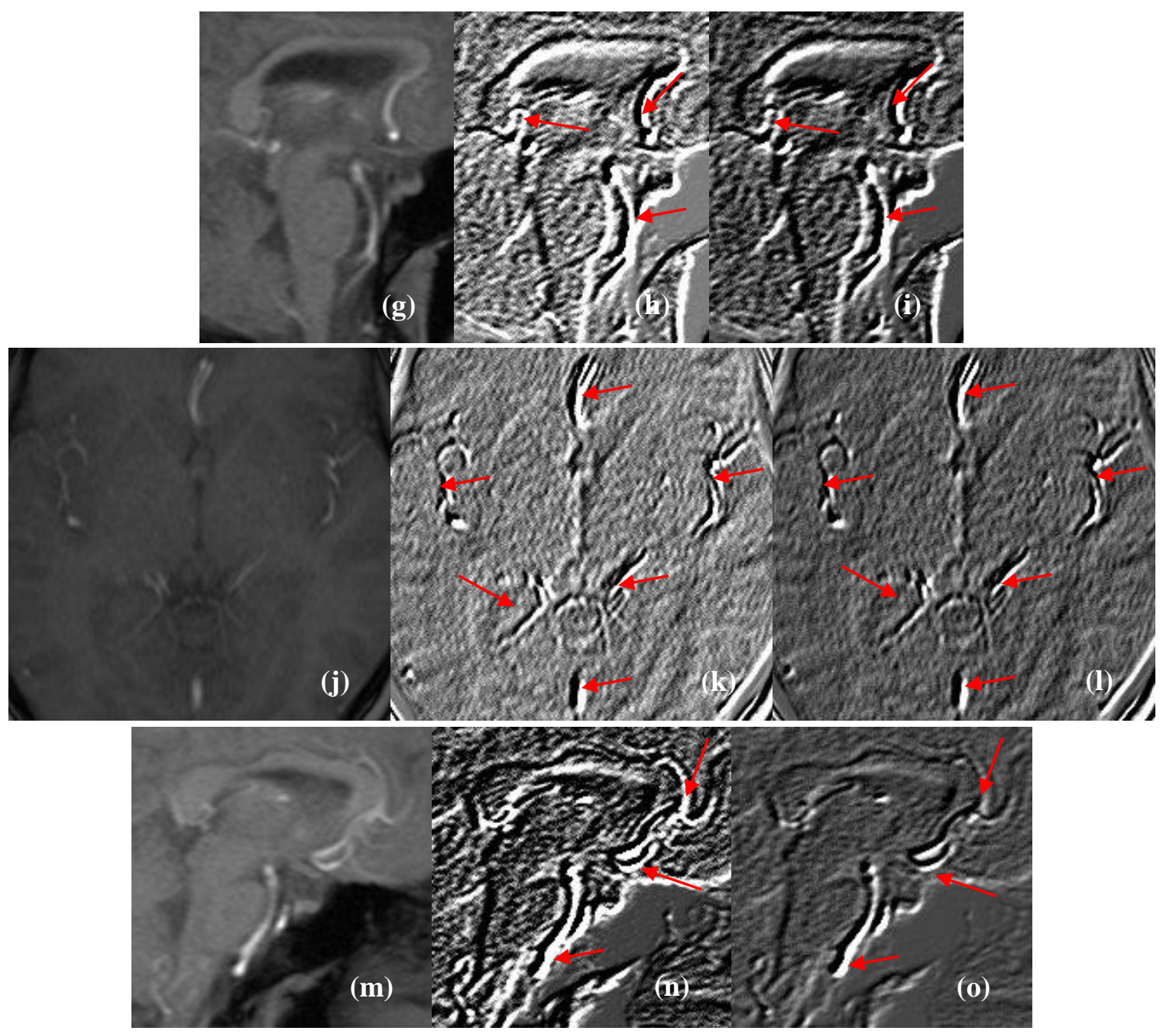

Fig. 4. MRI regions of interest are presented in $(a),(d),(g),(j)$ and $(m)$. The intensity-curvature term before interpolation $\left(E_{o}(x, y)\right)$ of the MRI is presented in $(b),(e),(h),(k)$ and $(n)$ respectively. The intensity-curvature term after interpolation $\left(E_{I N}(x, y)\right)$ is presented in $(c),(f),(i),(l)$ and $(o)$ respectively. Likewise Figure 3, the intensity-curvature terms were calculated using the bivariate cubic model function fitted to the MRI data. The arrows point to the human brain vessels.

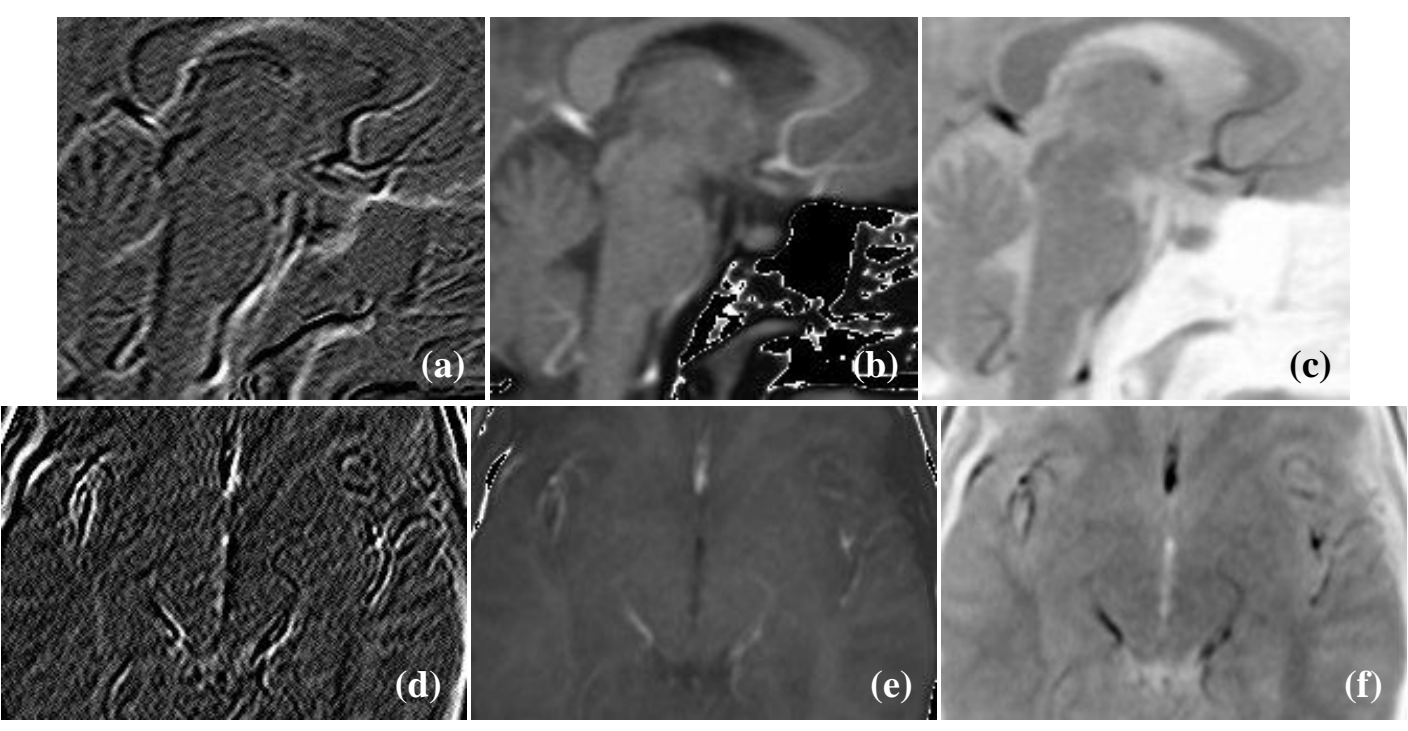



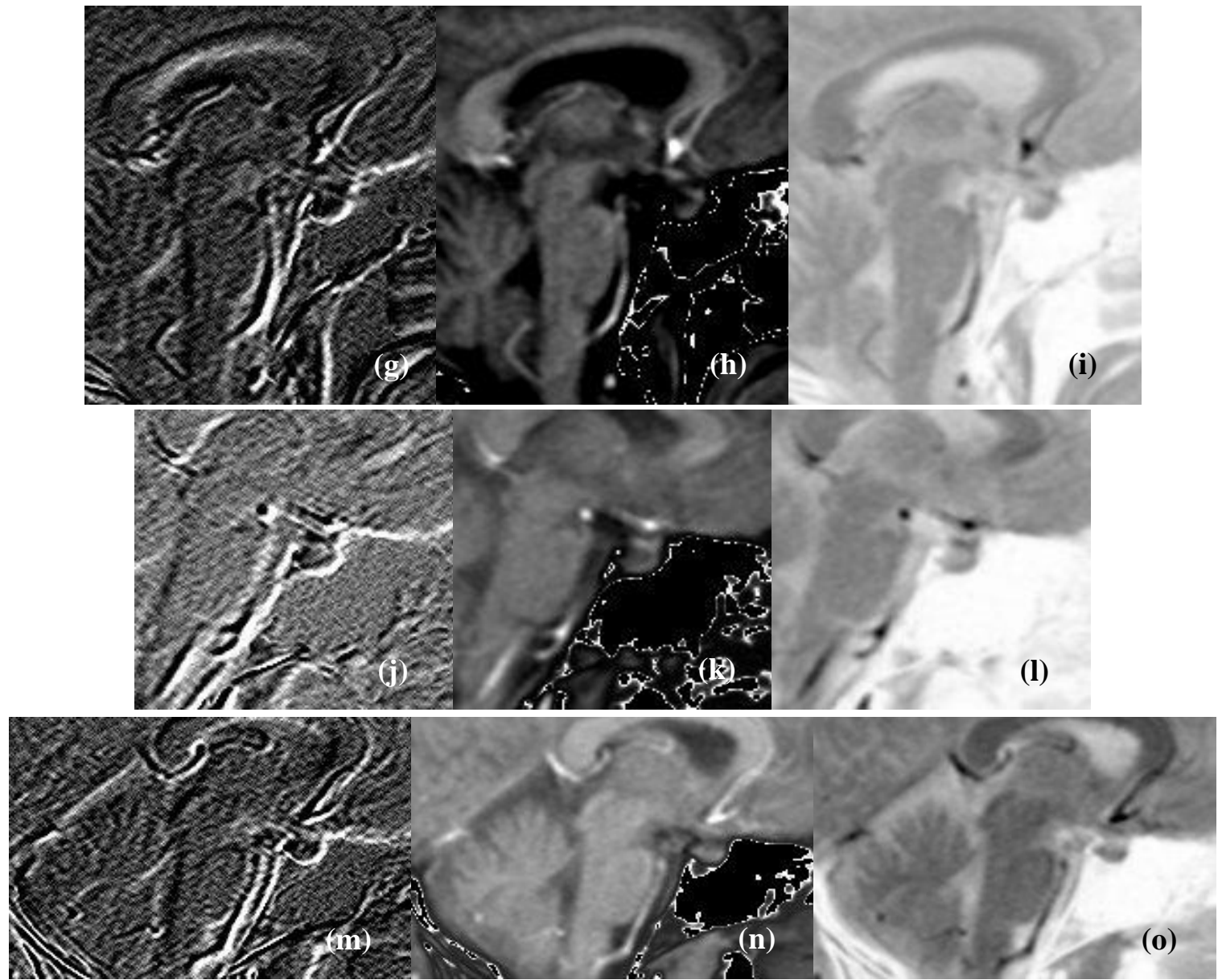

Fig. 5. the reference MRI images were presented in Figures $3 a, 3 d, 3 g, 3 j, 3 m$. In (a), (d), (g), (j) and (m) are presented the resultant $C C$ images. In $(b),(e),(h),(k)$ and $(n)$ are presented the resultant $S R I$ images. In $(c),(f),(i),(l)$ and $(o)$ are presented the resultant $R C$ images.

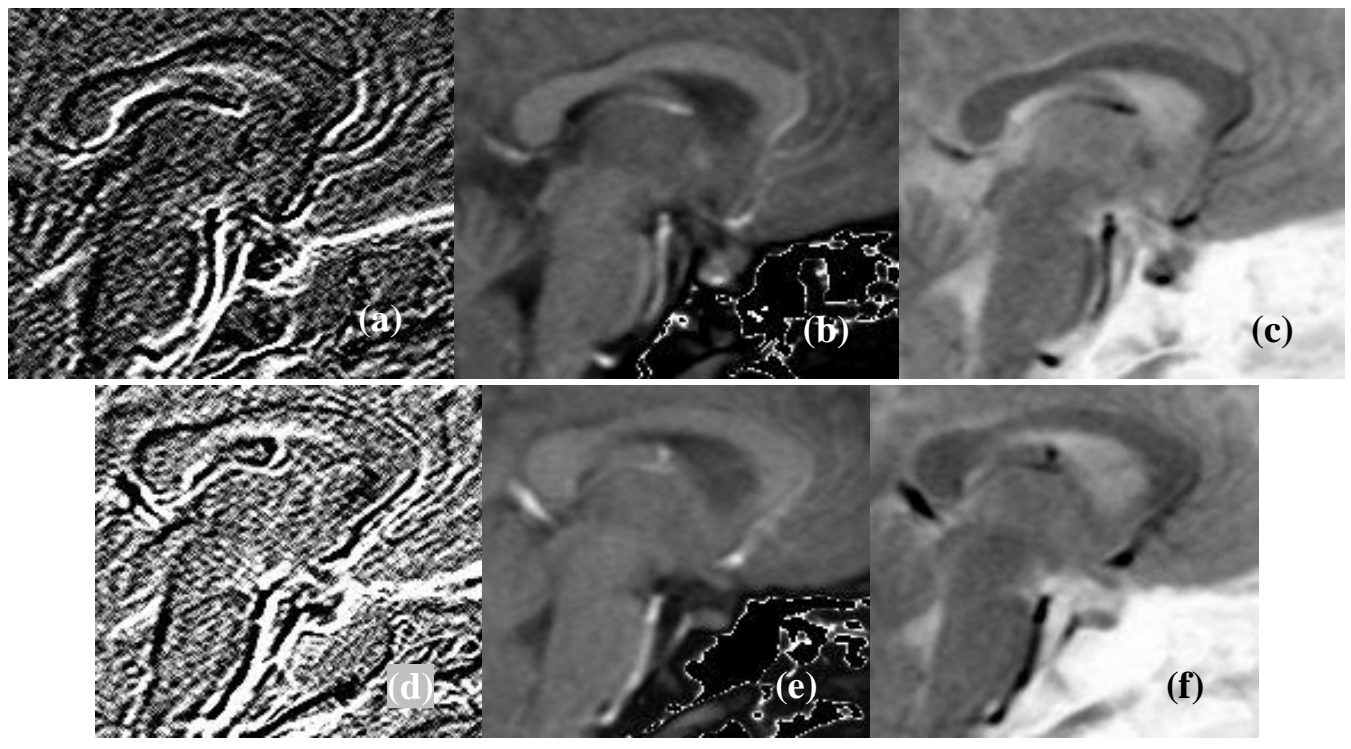


The Properties of the Intensity-Curvature Measurement Approaches...

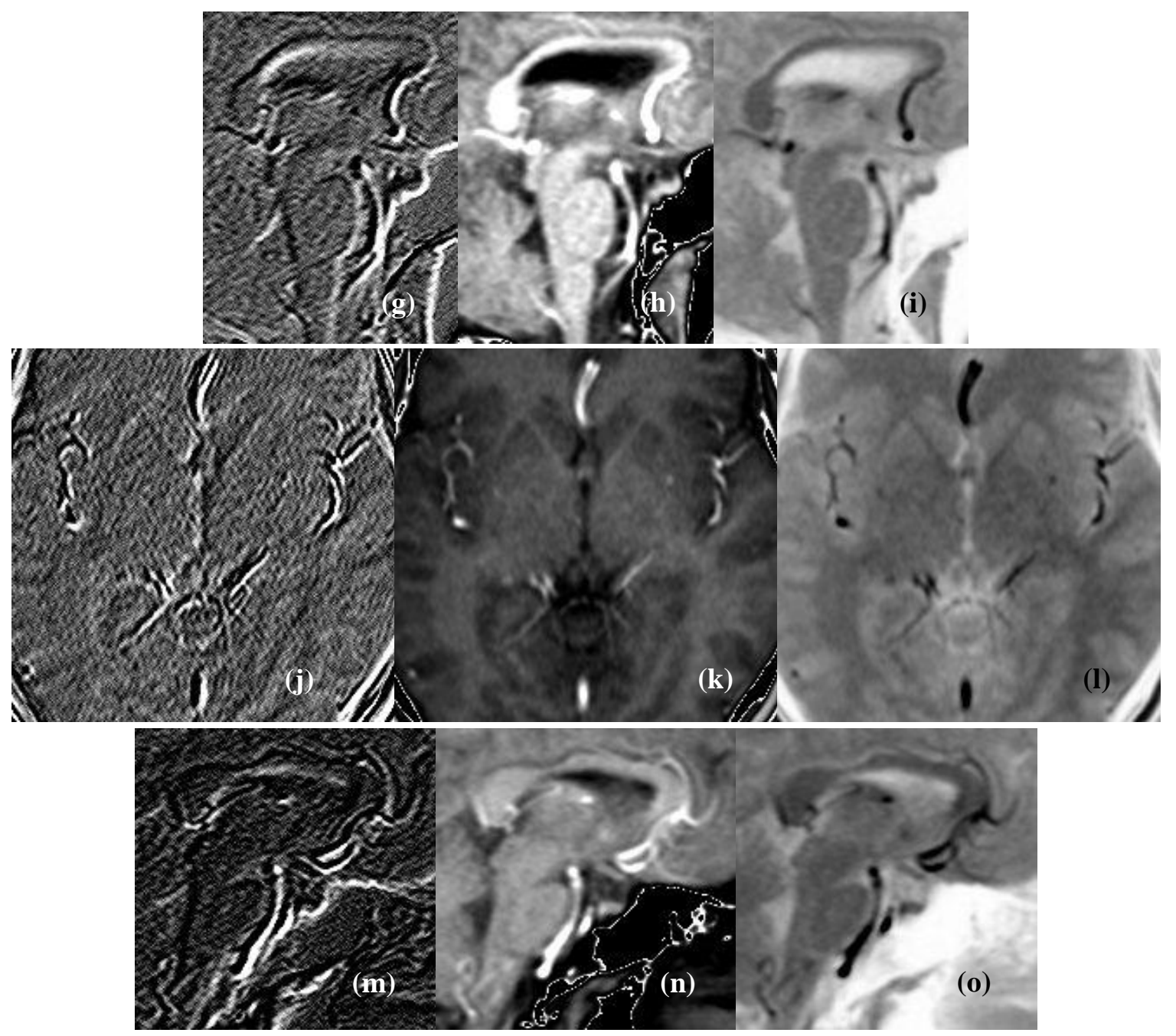

Fig. 6. The MRI regions of interest were presented in Figure 4 in $(a),(d),(g),(j)$ and $(m)$. The pictures presented here are the classic-curvature images: $(a),(d),(g),(j)$ and $(m)$. The signal resilient to interpolation images: $(b),(e),(h),(k)$ and $(n)$. And, the resilient curvature images in $(c),(f),(i),(l)$ and (o). Noteworthy is the remarkable highlight on the vasculature offered by the signal resilient to interpolation images in $(h),(k)$ and $(n)$.

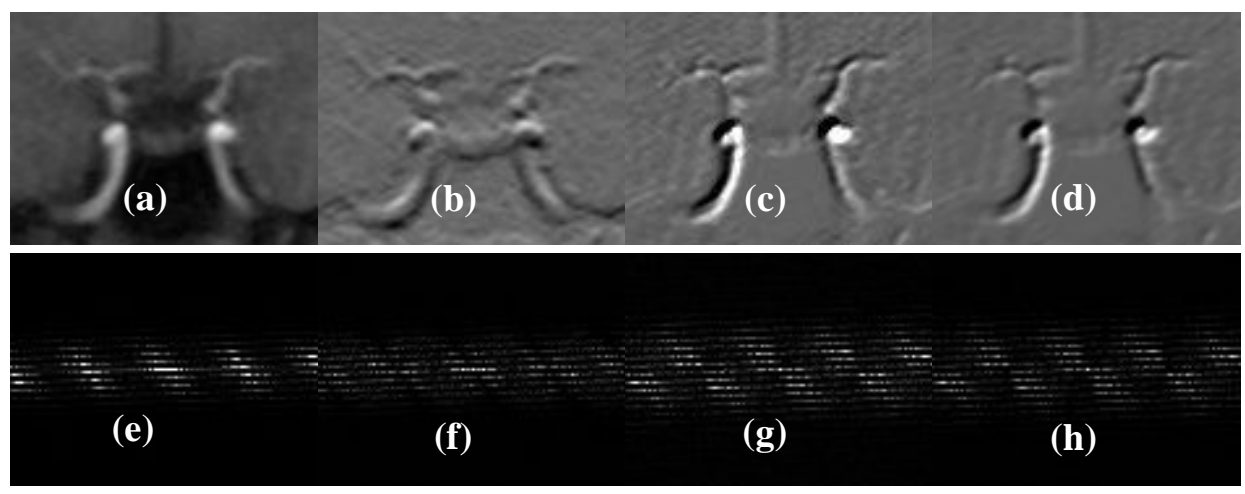

Fig. 7. The MRI image presented in (a) is high pass filtered and presented in $(b)$. The $E_{o}(x, y)$ and the $E_{I N}(x, y)$ of (a) were calculated from the bivariate cubic polynomial function and are presented in (c) and (d) respectively. The $k$-space magnitude of $(a)$ and $(b)$ is presented in $(e)$ and $(f)$ respectively. The $k$-space magnitude of $(c)$ and $(d)$ is presented in $(g)$ and $(h)$ respectively. 


\section{DISCUSSION}

MRI initial post-processing techniques had been under development and research since more than two decades now, and had released to the scientific community well reputable software for segmentation (Collins et al., 1995), registration and co-registration (Friston et al., 1995, Ashburner \& Friston 1997), spatial normalization (Ashburner \& Friston 1999, 2000), and filtering (Gerig et al., 1992). For an extended review of MRI postprocessing software the reader is referred to Cui et al. (2015). Feature extraction from MRI images is another important task in MRI post-processing and is accomplished here through the use of resampling techniques called ICMAs. Hence, to explain the properties of the $\mathrm{CC}$ and its outward show, it is due to mention that in order to calculate the $\mathrm{CC}$, the model polynomial function needs to fit the image data on a pixel by pixel basis. Thus, the CC calculates an additional image which outward show is the direct consequence of the mathematical function used to fit the image data (see Figures 1d, 1e, 1f, 1g, 1h, 1i). Consequential to the $\mathrm{CC}$ is the ICMA called intensity-curvature functional (ICF), which merges the image intensity with the $\mathrm{CC}$ (see for instance Figs. 1j, 1k, 1l). While studying human brain tumors the ICF showed the visually perceptible third dimension in proximity of the accumulation of fluids, next to the tumor mass (Ciulla et al., 2014). Moreover, the CC appears to be similar to the ICF, because (when calculated using the bivariate cubic model polynomial function) it also displays the visually perceptible third dimension (Ciulla et al., 2016a). This work presents evidence that the $\mathrm{CC}$ and the ICF are not quite the same as high pass

filtered signals. Moreover, the k-space of the $\mathrm{CC}$ is different from the k-space of the ICF (see Fig. 2). Recent research reports on the meaning and the nature of the ICMAs showing that: (i) the $\mathrm{CC}$ and the ICF are filter masks (Ciulla et al., 2016c), (ii) the signal resilient to interpolation is a filter (Ciulla et al., 2016b), and (iii) the resilient curvature inverts, smooths and magnifies (all at once) the grayscale of the image (Ciulla et al., 2016b). Samples of the SRI and the RC of MRI zones of interest are presented in figures 5 and 6 , and the MRIs are presented in figures 3 and 4 . Additional research yields the novelty presented in this paper. Two more images are derived from the ICF, and they are the intensity-curvature term before interpolation $\left(\mathrm{E}_{\mathrm{o}}(\mathrm{x}, \mathrm{y})\right)$, and the intensity-curvature term after interpolation $\left(\mathrm{E}_{\mathrm{IN}}(\mathrm{x}, \mathrm{y})\right)$. When calculated from the bivariate cubic model polynomial function, the intensity-curvature terms are similar to the CC. The similarity consists of the visually perceptible third dimension, which is prominent because of the presumed accumulation of fluids (Ciulla et al., 2016a). It can be therefore inferred that the visually perceptible third dimension of the $\mathrm{E}_{\mathrm{o}}(\mathrm{x}, \mathrm{y})$ and the $\mathrm{E}_{\mathrm{IN}}(\mathrm{x}, \mathrm{y})$ images might also be the indicator of fluids accumulation. And even so, though, the intensity-curvature term images are able to highlight the vessels of the human brain.

\section{CONCLUSION}

The intensity-curvature measurement approaches are image processing techniques which theoretical basis is the intensity-curvature concept. The notion is to merge together the image intensity with the sum of second order partial derivatives of the polynomial model function fitted to the image data. The aforementioned sum is called classic-curvature. The classic-curvature and the intensity-curvature functional are not quite the same as high pass filtered signal and this is elucidated through the study of their k-space magnitude. The classiccurvature and the intensity-curvature functional are mask images. The signal resilient to interpolation is an alternative filter. Likewise the resilient curvature, which moreover, inverts, smooths and magnifies the grayscale of an image. When calculated from the bivariate cubic model polynomial function, the two intensity-curvature terms show the visually perceptible third dimension very much alike the classic-curvature. The visually perceptible third dimension might be related to the accumulation of fluids. Hence, the intensitycurvature terms display the characteristic to highlight the human brain vasculature detected with MRI. Finally, the comparison between the k-space magnitude of the intensity-curvature terms of the MRI and the k-space of the high pass filtered signal of the MRI, reveals that ICTs are not the same as high pass filtered signal.

\section{REFERENCES}

Ashburner, J. (2012). SPM: a history. Neuroimage, 62: 791-800.

Ashburner, J. and Friston, K. J. (1997). Multimodal image co-registration and Partitioning - A unified framework. Neuroimage, 6: 209-217.

Ashburner, J. and Friston, K. J. (2000). Voxelbased morphometry - The methods. Neuroimage, 11: 805-821. 
Ashburner, J., and Friston, K. J. (1999). Nonlinear spatial normalization using basis functions. Human Brain Mapping, 7: 254-266.

Ciulla, C.; Rechkoska, U. S.; Capeska, D. B.; Risteski, F. A. and Veljanovski, D. (2014). On the intensity-curvature functional of the bivariate linear function: the third dimension of magnetic resonance 2D images in a tumor case study. American Journal of Signal Processing, 4: 41-48.

Ciulla, C.; Shikoska, U. R.; Veljanovski, D. and Risteki，F. A. (2018). Intensity-curvature highlight of human brain magnetic resonance imaging vasculature. Int. J. Modelling, Identification and Control, 29: XXX.

Ciulla, C.; Veljanovski, D.; Rechkoska, S. U. and Risteski F. A. (2015). Intensity-curvature measurement approaches for the diagnosis of magnetic resonance Imaging brain tumors. Journal of Advanced Research, 6: 10451069.

Ciulla, C.; Veljanovski, D.; Rechkoska, S. U. and Risteski, F. A. (2016a). On the properties of the intensity-curvature measurement approaches: the classic-curvature and the intensity-curvature functional. Journal of Solid Tumors, 6: 38-53.

Ciulla, C.; Veljanovski, D.; Rechkoska, S. U. and Risteski, F. A. (2016c). On the properties of the intensity-curvature measurement approaches: The signal resilient to interpolation and the resilient curvature. International Journal of Innovative Computing and Applications, 7: 91-118.

Ciulla, C.; Veljanovski, D.; Risteski, F. A. and Rechkoska, S. U. (2016b). On the filtering properties of classic-curvature and intensitycurvature functional images: Applications in magnetic resonance imaging. International Journal of Applied Pattern Recognition, 3: 77-98.

Collins, D. L.; Holmes, C. J.; Peters, T. M. and Evans, A. C. (1995). Automatic 3-D modelbased neuroanatomical segmentation. Human Brain Mapping, 3: 190-208.

Cox, R. W. (1996). AFNI: software for analysis and visualization of functional magnetic resonance neuroimages. Comput. Biomed. Res. 29: 162-173.

Cui, Z.; Zhao, C. and Gong, G. (2015). Parallel workflow tools to facilitate human brain MRI post-processing. Frontiers in Neuroscience, 9: 1-7.

Cutrer, F.; Michael, A.; Sorensen, G.; Weisskoff, R. M.; Østergaard, L.; Del Rio, M. S. et al. (1998). Perfusion weighted imaging defects during spontaneous migrainous aura. Annals of Neurology, 43: 25-31.

Dashjamts, T.; Yoshiura, T.; Hiwatashi, A.; Yamashita, K.; Abe, K.; Ohyagi, Y. et al. (2010). Asymmetrical cerebral perfusion demonstrated by noninvasive arterial spinlabeling perfusion imaging in a patient with corticobasal degeneration. Japanese Journal of Radiology, 28: 75-78.

Friston, K. J.; Ashburner, J.; Frith, C. D.; Poline, J. B.; Heather, J. D. and Frackowiak, R. S. J. (1995). Spatial registration and normalization of images. Human Brain Mapping, 3: 165189.

Gerig, G.; Kübler, O.; Kikinis, R. and Jolesz, F. (1992). Nonlinear anisotropic filtering of MRI data. IEEE Transactions on Medical Imaging, 11: 221-232.

Haacke, E. M.; Brown, R. F.; Thompson, M. and Venkatesan, R. (1999). Magnetic resonance imaging: Physical principles and sequence design. New York: J. Wiley \& Sons, USA.

Haacke, E. M.; Khan, M. A.; Manova, E. S.; Krishnamurthy, B.; Gollapalli, L.; Ciulla, C. et al. (2007). Establishing a baseline phase behavior in magnetic resonance imaging to determine normal vs. abnormal iron content in the brain. Journal of Magnetic Resonance Imaging, 26: 256-264.

Haacke, E. M.; Liu, S.; Buch, S.; Zheng, W.; Wu, D. and Ye, Y. (2015). Quantitative susceptibility mapping: current status and future directions. Magnetic Resonance Imaging, 33: 1-25.

Haacke, E. M.; Xu, Y.; Cheng, Y-C. N. and Reichenbach, J. R. (2004). Susceptibility weighted imaging (SWI). Magnetic Resonance in Medicine, 52: 612-618.

Hajnal, J. V.; Bryant, D. J.; Kabuboski, L.; Pattany, P. M.; De Coene, B.; Lewis, P. D. et al. (1992). Use of fluid attenuated inversion recovery (FLAIR) pulse sequences in MRI of the brain. Journal of Computer Assisted Tomography, 16: 841-844.

Isobe, T.; Yamamoto, T.; Akutsu, H.; Anno, I.; Shiigai, M.; Zaboronok, A. et al. (2010). Proton magnetic resonance spectroscopy 
findings of hemangioblastoma. Japanese Journal of Radiology, 28: 318-321.

Lauterbur, P. C. (1973). Image formation by induced local interactions: examples of employing nuclear magnetic resonance. Nature, 242:190-191.

Le Bihan, D.; Breton, E.; Lallemand, D.; Grenier, P.; Cabanis, E. and Laval-Jeantet, M. (1986). MR imaging of intra-voxel incoherent motions: Application to diffusion and perfusion in neurologic disorders. Radiology, 161: 401-407.

Mansfield, P. (1962). Proton magnetic resonance relaxation in solids by transient methods, $\mathrm{PhD}$ Dissertation. Queen Mary College, University of London, London, UK.
Ogawa, S.; Menon, R. S.; Tank, D. W.; Kim S. G.; Merkle, H.; Ellermann, J. M. et al. (1993). Functional brain mapping by blood oxygenation level-dependent contrast magnetic resonance imaging. A comparison of signal characteristics with a biophysical model. Biophysical Journal, 64: 803-812.

Pierpaoli, C.; Jezzard, P.; Basser, P. J.; Barnett, A. and Di Chiro, G. (1996). Diffusion tensor MR imaging of the human brain. Radiology, 201: 637-648.

Rauscher, A.; Sedlacik, J.; Barth, M.; Mentzel, H. J. and Reichenbach, J. R. (2005). Magnetic susceptibility-weighted MR phase imaging of the human brain. American Journal of Neuroradiology, 26: 736-742. 